Journal of Mathematics and Statistics 8 (2): 211-215, 2012

ISSN 1549-3644

(C) 2012 Science Publications

\title{
Fuzzy Orders in the Real Line
}

\author{
${ }^{1}$ Lemnaouar Zedam and ${ }^{2}$ Abdelkader Stouti \\ ${ }^{1}$ Department of Mathematics, Faculty of Mathematics and Informatics, \\ Laboratory of Pure and Applied Mathematics, M'sila University, M'sila 28105, Algeria \\ ${ }^{2}$ Laboratory of Mathematics and Applications, Faculty of Sciences and Techniques, \\ University Sultan Moulay Slimane, P.O. Box 523, 23000 Beni-Mellal, Morocco
}

\begin{abstract}
Problem statement: In this study, we characterize a subcategory of fuzzy orders which were compatible with the usual addition and the multiplication by scalar on the real line R. Results and Conclusion: All possible forms of fuzzy orders of this subcategory are given.
\end{abstract}

Key words: Fuzzy set, fuzzy relation, fuzzy order, linear fuzzy order, usual addition, multiplication

\section{INTRODUCTION}

The theory of fuzzy order relations which is a generalization of the concept of crisp order relations first was introduced by Zadeh (1971) and then many researchers have been working on this new field and developed it (see for example, (Beg, 2004; BouchonMeunier, 1995; Billot, 1992; Bodenhofer, 2003; Bodenhofer et al., 2007; Dubois and Prade, 1980; Gerla and Crisconio, 2001; Kaufmann and Zadeh, 1973; Kundu, 2000; Ovchinnikov, 1991; Stouti, 2003; Stouti and Zedam, 2010; Venugopalan, 1992; Zimmermann, 2001). In this study, we are interesting to study fuzzy orders on real line $\mathbb{R}$.

This study is organized as follows. We recall some well know definitions and we give some examples of fuzzy orders on real line $\mathbb{R}$. Next, we shall give a theorem which will provide us the general form of fuzzy orders which are compatible with the usual addition and multiplication on the real line $\mathbb{R}$ (see Theorem 3.6). We finish by a theorem shows that the converse of Theorem 3.6 is not necessarily true.

Preliminary definitions: Let $X$ be a universe of discourse. A fuzzy subset $\mathrm{A}$ of $X$ defined by Zadeh (1965) is characterized by a membership function A: $\mathrm{X} \rightarrow[0,1]$, where $\mathrm{A}(\mathrm{x})$ is interpreted as the degree of membership of the element $x$ in the fuzzy subset A for each $\mathrm{x} \in \mathrm{X}$.

Definition 2.1 (Zadeh, 1971): Let $X$ be a nonempty set. A fuzzy relation $r$ on $X$ is a function $r: X \times X \rightarrow[0$, 1]. For every $x, y \in X$, the value $r(x, y)$ is called the grade of membership of $(\mathrm{x}, \mathrm{y})$ in $\mathrm{r}$ and means how far $\mathrm{x}$ and $\mathrm{y}$ are related under $\mathrm{r}$.

A crisp binary relation $r$ is a particular fuzzy relation where the real interval $[0,1]$ of scalars is replaced by the set $\{0,1\}$ of integers, that is $r(x, y)=0$ or $\mathrm{r}(\mathrm{x}, \mathrm{y})=1$. For more details we refer to (BouchonMeunier, 1995; Kaufmann and Zadeh, 1973; Zimmermann, 2001).

Zadeh (1971), gave the following definition of fuzzy order.

Definition 2.2 (Zadeh, 1971): Let $X$ be a nonempty set. A fuzzy order on $X$ is a fuzzy relation $\mathrm{r}$ in $\mathrm{X}$ satisfying the following three properties:

- For all $\mathrm{x} \in \mathrm{X}, \mathrm{r}(\mathrm{x}, \mathrm{x})=1$ (fuzzy reflexivity)

- For all $x, y \in X, x \neq y$ and $r(y, x)>0$ implies $r(y$, $\mathrm{x})=0$, (fuzzy antisymmetry)

- For all $x, z \in X, \quad r(x, z) \geq \sup _{y \in X}[\min \{r(x, y), r(y, z)\}]$,

(fuzzy transitivity)

A nonempty set $X$ with a fuzzy order $r$ defined on it is called fuzzy ordered set (foset, for short) and we denote it by $(\mathrm{X}, \mathrm{r})$. If $Y$ is a subset of a foset $(\mathrm{X}, \mathrm{r})$, then the fuzzy order $r$ is a fuzzy order on $\mathrm{Y}$ and is called the induced fuzzy order.

A fuzzy order $r$ is linear (or total) on $\mathrm{X}$ if for every $\mathrm{x}, \mathrm{y} \in \mathrm{X}$ we have $\mathrm{r}(\mathrm{x}, \mathrm{y})>0$ or $\mathrm{r}(\mathrm{y}, \mathrm{x})>0$. A fuzzy ordered set $(X, r)$ in which $r$ is total is called a $r$-fuzzy chain. Conversely, if for any $x, y \in X, r(x, y)>0$ if and only if $\mathrm{x}=\mathrm{y}$ then $(\mathrm{X}, \mathrm{r})$ is called $\mathrm{r}$-fuzzy antichain.

Corresponding Author: Lemnaouar Zedam, Department of Mathematics, Faculty of Mathematics and Informatics, Laboratory of Pure and Applied Mathematics, M'sila University, M'sila 28105, Algeria 
Let $(\mathrm{X}, \mathrm{r})$ be a fuzzy ordered set and $\mathrm{A}$ be a subset of X:

- An element $u \in X$ is a r-upper bound of $A$ if $r(x, u)$ $>0$ for all $x \in A$. If $u$ is $r$-upper bound of $A$ and $u$ $\in A$ then $u$ is called the greatest element of $A$. The r-lower bound and the least element are defined analogously

- An element $m \in A$ is called a maximal element of $A$ if $r(m, x)>0$ for some $x \in A$ then $m=x$. Minimal elements are defined similarly

- An element $s \in X$ is the r-supremum of $A$ if $s$ is $r-$ upper bound of $\mathrm{A}$ and for all $\mathrm{r}$-upper bound $\mathrm{u}$ of $\mathrm{A}$, we have $r(s, u)>0$. When $s$ exists, we shall write $s$ $=\sup _{\mathrm{r}}(\mathrm{A})$. Similarly, $1 \in \mathrm{X}$ is the $\mathrm{r}$-infimum of $\mathrm{A}$ if 1 is a r-lower bound of $\mathrm{A}$ and for all $\mathrm{r}$-lower bound $\mathrm{k}$ of $\mathrm{A}$, we have $\mathrm{r}(\mathrm{k}, \mathrm{l})>0$. When 1 exists we shall write $l=\inf _{\mathrm{r}}(\mathrm{A})$

Next, we shall give some examples of fuzzy partial and linear (or total) orders.

- $\quad$ Let $X=\{a, b, c, d, e, f, g\}$. Then the fuzzy subset $r$ defined on $\mathrm{X} \times \mathrm{X}$ by the following Table 1 is a fuzzy order on $X$

- Let $X=\mathbb{R}$ and $\lambda>0$ Then, the fuzzy relation $r_{\lambda}$ defined for all $\mathrm{x}, \mathrm{y} \in \mathrm{X}$ by:

$$
r_{\lambda}(x, y)=\left\{\begin{array}{c}
1, \text { if } x=y \\
\min \left(1, \frac{y-x}{\lambda}\right), \quad \text { if } x<y \\
0, \quad \text { if } x>y
\end{array}\right.
$$

is a linear fuzzy order on $\mathbb{R}$.

- Let $X=\mathbb{R}$. Then, the fuzzy relation $r$ defined for all $\mathrm{x}, \mathrm{y} \in \mathrm{X}$ by:

$$
r(x, y)=\left\{\begin{array}{c}
1, \text { if } x=y \\
0, \text { if } x>y \\
1-\frac{x}{y}, \text { if } 0 \leq x<y \\
1-\frac{y}{x}, \text { if } x<y \leq 0 \\
1, \text { if } x<0 \text { and } y>0
\end{array}\right.
$$

is a linear fuzzy order on $\mathbb{R}$.

Table 1: Fuzzy order on $\mathrm{X}$

\begin{tabular}{lllllc}
\hline $\mathrm{r}(.,)$. & $\mathrm{a}$ & $\mathrm{b}$ & $\mathrm{c}$ & $\mathrm{d}$ & $\mathrm{e}$ \\
\hline $\mathrm{a}$ & 1 & 0 & 0 & 0.65 & 0.40 \\
$\mathrm{~b}$ & 0 & 1 & 0 & 0.35 & 0.45 \\
$\mathrm{c}$ & 0 & 0 & 1 & 0.00 & 0.70 \\
$\mathrm{~d}$ & 0 & 0 & 0 & 1.00 & 0.00 \\
$\mathrm{e}$ & 0 & 0 & 0 & 0.00 & 1.00 \\
\hline
\end{tabular}

Characterization of a subcategory of fuzzy orders which are compatible with the structures of the real line $\mathbb{R}:$ We shall give a characterization (or the possible forms) of a subcategory of fuzzy orders which are compatible with the usual addition and the multiplication by scalar on the real line $\mathbb{R}$ (Theorem 3.7). First, we need the following definitions.

\section{Definition 3.1:}

- We say that $r$ is compatible with the addition if for all $\left(\mathrm{x}_{1}, \mathrm{y}_{1}\right),\left(\mathrm{x}_{2}, \mathrm{y}_{2}\right) \in \mathbb{R}^{2}$, we have $\mathrm{r}\left(\mathrm{x}_{1}, \mathrm{y}_{2}\right)>0$ and $\mathrm{r}\left(\mathrm{x}_{2}, \mathrm{y}_{2}\right)>0 \Rightarrow \mathrm{r}\left(\mathrm{x}_{1}+\mathrm{x}_{2}, \mathrm{y}_{1}+\mathrm{y}_{2}\right)>0$

- The fuzzy order $r$ is said to be compatible with the multiplication by scalar if for all $(x, y) \in \mathbb{R}^{2}$ and $\lambda>0$, we have $\mathrm{r}(\mathrm{x}, \mathrm{y})>0 \Rightarrow \mathrm{r}(\lambda \mathrm{x}, \lambda \mathrm{y})>0$

Next we shall define a subcategory of fuzzy orders which are compatible with the addition and the multiplication by scalar on the real line. More precisely, we consider the following.

Definition 3.2: Let $\mathbb{P}$ the set of all fuzzy orders defined on $\mathbb{R}$ satisfying the two following conditions:

- For all $\left(\mathrm{x}_{1}, \mathrm{y}_{1}\right),\left(\mathrm{x}_{2}, \mathrm{y}_{2}\right) \in \mathbb{R}^{2,}$ we have $\mathrm{r}\left(\mathrm{x}_{1}+\mathrm{x}_{2}, \mathrm{y}_{1}+\mathrm{y}_{2}\right) \geq \min \left\{\mathrm{r}\left(\mathrm{x}_{1}, \mathrm{y}_{1}\right), \mathrm{r}\left(\mathrm{x}_{2}, \mathrm{y}_{2}\right)\right\}$

- For all $(x, y) \in \mathbb{R}^{2}$ and $\lambda>0$, we have $\mathrm{r}(\lambda \mathrm{x}, \lambda \mathrm{y}) \geq \mathrm{r}(\mathrm{x}, \mathrm{y})$.

Proposition 3.3: Let $r$ be an element of $\mathbb{P}$. Then, $r$ is compatible with the addition and the multiplication by scalar on the real line $\mathbb{R}$.

Proof: Let $r$ be a fuzzy order such that $r \in \mathbb{P}$.

Let $\left(\mathrm{x}_{1}, \mathrm{y}_{1}\right),\left(\mathrm{x}_{2}, \mathrm{y}_{2}\right) \mathbb{R}^{2}$ such that $\mathrm{r}\left(\mathrm{x}_{1}, \mathrm{y}_{1}\right)>0$ and $r\left(x_{2}, y_{2}\right)>0$. Since $r \in \mathbb{P}$ it then follows that:

$$
\mathrm{r}\left(\mathrm{x}_{1}+\mathrm{x}_{2}, \mathrm{y}_{1}+\mathrm{y}_{2}\right) \geq \min \left\{\mathrm{r}\left(\mathrm{x}_{1}, \mathrm{y}_{1}\right), \mathrm{r}\left(\mathrm{x}_{2}, \mathrm{y}_{2}\right)\right\}
$$

Hence:

$$
\mathrm{r}\left(\mathrm{x}_{1}+\mathrm{x}_{2}, \mathrm{y}_{1}+\mathrm{y}_{2}\right)>0
$$

Thus, $\mathrm{r}$ is compatible with the addition.

Let $(x, y) \in \mathbb{R}^{2}$ and $\lambda>0$ such that $r(x, y)>0$. Since $r \in \mathbb{P}$ it holds that $r(\lambda x, \lambda y) \geq r(x, y)>0$.

Hence: 


$$
r(\lambda x, \lambda y)>0
$$

Thus, $r$ is compatible with the multiplication by scalars.

The following example shows that the set of all fuzzy orders which are compatible with the addition and the multiplication by scalar on $\mathbb{R}$ is not equal to $\mathbb{P}$.

Let us consider the fuzzy order $r$ defined on $\mathbb{R}$ given in the above example. For all $\mathrm{x}, \mathrm{y} \in \mathbb{R}$ :

$$
r(x, y)=\left\{\begin{array}{c}
1, \text { if } x=y \\
0, \text { if } x>y ; \\
1-\frac{x}{y}, \text { if } 0 \leq x<y \\
1-\frac{y}{x}, \text { if } x<y \leq 0 ; \\
1, \text { if } x<0 \text { and } y>0
\end{array}\right.
$$

$r$ is compatible with the addition and the multiplication by scalar on $\mathbb{R}$. But, $r \notin \mathbb{P}$. Indeed, we have $r(0,1)=1, \quad r(1,1)=1$ and $r(1,2)=\frac{1}{2}$. We have also, $\quad(1,2)=(0,1)+(1,1), \quad$ but $\mathrm{r}(0+1,1+1)=\frac{1}{2}<\min \{\mathrm{r}(0,1), \mathrm{r}(1,1)\}=1$.

Proposition 3.4: Let $r$ be an element of $\mathbb{P}$. Then, for all $\mathrm{x}, \mathrm{y} \in \mathbb{R}$ we have:

$$
r(x, y)=r(x-y, 0)=r(0, y-x)
$$

\section{Proof: Let $\mathrm{x}, \mathrm{y} \in \mathbb{R}$ :}

- First let us show that: $r(x, y)=r(0, y-x)$. Indeed, since $(x, y)=(0, y-x)+(x, x)$ then $r(x, y)=r((0$, $y-x)+(x, x))$. On the other hand, since $r \in \mathbb{P}$, it then follows that $r(x, y) \geq \min \{r(0, y-x), r(x, x)\}$. As $r(x, x)=1$, it holds that $r(x, y) \geq r(0, y-x)$. Conversely, $r(0, y-x)=r((x, y)+(-x,-x)$. So, we have $r(0, y-x) \geq \min \{r(x, y), r(-x,-x)\}$. In the same way, as $\mathrm{r}(-\mathrm{x},-\mathrm{x})=1$, it holds that $r(x, y) \leq r(0, y-x)$. Therefore, $r(x, y)=r(0, y-x)$

- We have $r(x, y)=r(x-y, 0)$. Indeed, since $(x, y)=$ $(x-y, 0)+(y, y)$ then $r(x, y)=r((x-y, 0)+(y, y))$. Hence, $r(x, y) \geq \min \{r(x-y, 0), r(y, y)\}$. Thus, we have $r(x, y) \geq r(x-y, 0)$. On the other hand, we have $r(x-y, 0)=r((x, y)+(-y,-y))$. Hence, since $r \in \mathbb{P}$ we get $r(x-y, 0) \geq \min \{r(x, y), r(y, y)\}$. Then, we obtain $r \quad(x-y, 0) \geq \quad r \quad(x, y)$. Thus, $\mathrm{r}(\mathrm{x}, \mathrm{y})=\mathrm{r}(\mathrm{x}-\mathrm{y}, 0)$
Consequentely, for all $\mathrm{x}, \mathrm{y} \in \mathbb{R}$ :

$$
r(x, y)=r(x-y, 0)=r(0, y-x)
$$

Definition 3.5: Let $r$ be a fuzzy order on $\mathbb{R}$ and $x \in \mathbb{R}$. If $\mathrm{r}(0, \mathrm{x})>0$, then $x$ is called a r-positive real number. The set of all $r$-positive real numbers is denoted by $\mathbb{R}_{\mathrm{r}}^{+}$. Similarly, if $\mathrm{r}(\mathrm{x}, 0)>0$, then $x$ is called a $r$-negative real number and the set of all $r$-negative real numbers is denoted by $\mathbb{R}_{\mathrm{r}}^{-}$.

In order to proof the main result of this study (Theorem 3.7) we need to show the following Lemma.

Lemma 3.6: Let $r$ be an element of $\mathbb{P}$. Then, we have:

- For all $x, y \in \mathbb{R}$, if $x<y$, then $r(x, y)=r(0,1)$

- For all $x, y \in \mathbb{R}$, if $x>y$, then $r(x, y)=r(1,0)$

- $\mathbb{R}_{\mathrm{r}}^{+} \cap \mathbb{R}_{\mathrm{r}}^{-}=\{0\}$

- If $r$ is a total fuzzy order on $\mathbb{R}$, then $\mathbb{R}_{r}^{+} \cup \mathbb{R}_{r}^{-}=\mathbb{R}$

Proof: Let $r$ be an element of $\mathbb{P}$.

Let $\mathrm{x}, \mathrm{y} \in \mathbb{R}$ such that $\mathrm{x}<\mathrm{y}$. By Proposition 3.4, we have $r(x, y)=r(0, y-x)$. Since $r$ is compatible with the multiplication by scalar and $y-x>0$, it holds that $r(0, y-x) \geq r(0,1)$. On the other hand, we know that $r(0,1)=r\left(0, \frac{1}{y-x}(y-x)\right) \geq r(0, y-x) . \quad$ Therefore, $r(0,1)=r(0, y-x)=r(x, y)$.

- Follows in the same way.

- Let $x \in \mathbb{R}_{\mathrm{r}}^{+} \cap \mathbb{R}_{\mathrm{r}}^{-}$. Then, $\mathrm{x} \in \mathbb{R}_{\mathrm{r}}^{+}$and $\mathrm{x} \in \mathbb{R}_{\mathrm{r}}^{-}$. So, $\mathrm{r}(0$, $\mathrm{x})>0$ and $\mathrm{r}(\mathrm{x}, 0)>0$. Now, by fuzzy antisymmetry we get that $\mathrm{x}=0$. Hence, $\mathbb{R}_{\mathrm{r}}^{+} \cap \mathbb{R}_{\mathrm{r}}^{-} \subseteq\{0\}$

On the other hand, as $r(0,0)=1>0$ so $0 \in \mathbb{R}_{\mathrm{r}}^{+} \cap \mathbb{R}_{\mathrm{r}}^{-}$. Therefore, $\mathbb{R}_{\mathrm{r}}^{+} \cap \mathbb{R}_{\mathrm{r}}^{-}=\{0\}$

- Assume that $r$ is total on $\mathbb{R}$. So, for ever $x \in \mathbb{R}$ we have $r(0, x)>0$ or $r(x, 0)>0$. This implies that $\mathrm{x} \in \mathbb{R}_{\mathrm{r}}^{+}$or $\mathrm{x} \in \mathbb{R}_{\mathrm{r}}^{-}$. Hence, $\mathbb{R} \subseteq \mathbb{R}_{\mathrm{r}}^{+} \cap \mathbb{R}_{\mathrm{r}}^{-}$and therefore $\mathbb{R}=\mathbb{R}_{\mathrm{r}}^{+} \cap \mathbb{R}_{\mathrm{r}}^{-}$

Theorem 3.7: Let $r$ be an element of $\mathbb{P}$. Then, $r$ is defined for all $\mathrm{x}, \mathrm{y} \in \mathbb{R}$ as the following.

$$
\begin{aligned}
& \text { If } r(0,1)>0 \text { and } r(1,0)=0 \text {, then: } \\
& \qquad r(x, y)=\left\{\begin{array}{c}
1 \text { if } x=y \\
r(0,1) \text { if } x>y \\
0 \text { if } x<y
\end{array}\right.
\end{aligned}
$$


If $r(0,1)=0$ and $r(1,0)>0$, then:

$$
r(x, y)=\left\{\begin{array}{c}
1 \text { if } x=y \\
0 \text { if } x>y \\
r(1,0) \text { if } x<y
\end{array}\right.
$$

If $\mathrm{r}(0,1)=0$ and $\mathrm{r}(1,0)=0$, then:

$$
r(x, y)=\left\{\begin{array}{lll}
1 & \text { if } & x=y \\
0 & \text { if } & x \neq y
\end{array}\right.
$$

Proof: Let $x, y \in \mathbb{R}$ and $r \in \mathbb{P}$. We can distinguish three cases.

First case: If $x=y$, then by fuzzy reflexivity we get $\mathrm{r}(\mathrm{x}, \mathrm{y})=1$.

Second case: If $\mathrm{x}<\mathrm{y}$, it then follows from Lemma 3.6 that $r(x, y)=r(0,1)$.

Third case: If $\mathrm{x}<\mathrm{y}$, it then follows from Lemma 3.6 that $r(x, y)=r(1,0)$.

Hence, if $\mathrm{x} \neq \mathrm{y}$ we have either $\mathrm{x}<\mathrm{y}$ or $\mathrm{x}>\mathrm{y}$. This means that either $y>x$ or $x>y$. Thus, we have either $\mathrm{R}(\mathrm{x}, \mathrm{y})=\mathrm{r}(0,1)$ which implies $\mathrm{r}(\mathrm{y}, \mathrm{x})=\mathrm{r}(1,0)$ or $(\mathrm{r}(\mathrm{x}$, $y)=r(1,0)$ which implies $r(y, x)=r(0,1)$.

Now, by the fuzzy ant symmetry of $r$ we cannot have at the same time that $r(0,1) \neq 0$ and $r(1,0) \neq 0$. Therefore we get three possible forms of the fuzzy order $r \in \mathbb{P}$.

First form: If $\mathrm{r}(0,1)>0$ and $\mathrm{r}(1,0)=0$. Then:

$$
r(x, y)=\left\{\begin{array}{c}
1 \text { if } x=y \\
r(0,1) \text { if } x>y \\
0 \text { if } x<y
\end{array}\right.
$$

Second form: If $r(0,1)=0$ and $r(1,0)>0$. Then:

$$
r(x, y)=\left\{\begin{array}{c}
1 \text { if } x=y \\
0 \text { if } x>y \\
r(1,0) \text { if } x<y
\end{array}\right.
$$

Third form: If $r(0,1)=0$ and $r(1,0)=0$. Then:

$$
r(x, y)=\left\{\begin{array}{lll}
1 & \text { if } & x=y \\
0 & \text { if } & x \neq y
\end{array}\right.
$$

Note that if $r$ take the third form, then $(\mathbb{R}, r)$ is a $r-$ fuzzy antichain.
As consequences of Theorem 3.7, we obtain the following corollary:

Corollary 3.8: Let $r$ be an element of $\mathbb{P}$. Then, we have $r$ is total on $\mathbb{R}$ if and only if $r(1,0)>0$ or $r(1,0)>0$.

Conversely, we have the following result.

Theorem 3.9: Each fuzzy order in $\mathbb{R}$ takes one of the three forms given in theorem 3.7 isn't necessarily an element of $\mathbb{P}$.

Proof: Immediate.

\section{REFERENCES}

Beg, I., 2004. Some applications of fuzzy ordered relations, CUBO, 6: 103-114.

Billot, A., 1992. Economic theory of Fuzzy Equilibria: an Axiomatic Analysis. 1st Edn., Springer-Verlag, Berlin, ISBN: 354054982X, pp: 164.

Bodenhofer, U., 2003. Representations and constructions of similarity-based fuzzy orderings. Fuzzy Sets Syst., 137: 113-136. DOI: 10.1016/S0165-0114 (02)00436-0

Bodenhofer, U., B.D. Baets and J. Fodor, 2007. A compendium of fuzzy weak orders: Representations and constructions. Fuzzy Sets Syst., 158: 811-829. DOI: 10.1016/j. fuss. 2006.10.005

Bouchon-Meunier, B.M., 1995. La Logique Floue et ses Applications. 1st Edn., Addison-Wesley France, Paris, ISBN: 2879080738, pp: 257.

Dubois, D. and H.M. Prade, 1980. Fuzzy Sets and Systems: Theory and Applications. 4th Edn., Academic Press, New York, ISBN: 0122227506, pp: 393.

Gerla, G. and C. Crisconio, 2001. Fuzzy orders in approximate reasoning. Department of Mathematics and Computer Science.

Kaufmann, A. and L.A. Zadeh, 1973. Introduction a la Théorie des Sous-Ensembles Flous a L'usage Des Ingénieurs. 1st Edn., Masson, Paris, ISBN: 2225366306, pp: 410.

Kundu, S., 2000. Similarity relations, fuzzy linear orders and fuzzy partial orders. Fuzzy Sets Syst., 109: 419-428. DOI: 10.1016/S0165-0114 (97)00370-9

Ovchinnikov, S., 1991. Similarity relations, Fuzzy partitions and fuzzy ordering. Fuzzy Sets Syst., 40: 107-126. DOI: 10.1016/0165-0114 (91)90048-U

Stouti, A. and L. Zedam, 2010. On $\alpha$-fuzzy orders. J. Fuzzy Math., 18: 171-192. 
Stouti, A., 2003. Fixed point theory for fuzzy monotone multifunctions. J. Fuzzy Math., 11: 455-466.

Venugopalan, P., 1992. Fuzzy ordered sets. Fuzzy Sets Syst., 46: 221-226. DOI: 10.1016/0165-0114 (92)90134-P

Zadeh, L.A., 1965. Fuzzy sets. Inform. Control, 8: 338353. DOI: 10.1016/S0019-9958 (65)90241-X
Zadeh, L.A., 1971. Similarity relations and fuzzy orderings. Inform. Sci., 3: 177-200. DOI: 10.1016/S0020-0255 (71)80005-1

Zimmermann, H.J., 2001. Fuzzy Set Theory--and Its Applications. 4th Edn., Springer, Boston, ISBN: 9780792374350, pp: 514. 